\title{
EXPERIMENTAL DETERMINATION OF REFLECTION PHASES BY THREE-BEAM DIFFRACTION AND ITS APPLICATIONS
}

\author{
K. HÜMMER, W. SChWEgle AND E. WeCKert \\ Institut für Kristallographie, Universität Karlsruhe (TH) \\ Kaiserstr. 12, D(W)-7500 Karlsruhe, Germany
}

\begin{abstract}
Information on phase relationships between Bragg reflections can be obtained by the interference of Bragg waves. In the three-beam case, phase difference is given by a structure invariant triplet phase relationship. The intensity variation due to the three-beam interaction can be best measured by a $\psi$-scan experiment. The resulting $\psi$-scan diffraction profiles scanning through a three-beam position uniquely depend on the triplet phase relationship involved. In principle each three-beam profile is given by a superposition of a symmetrical phase-independent and a phase-dependent profile. Thus, triplet phases can be determined experimentally with an accuracy of about 45 degrees. The $\psi$-scan method is discussed and some examples of applications to the determination of the absolute structure as well as to the structure determination by combination of measured triplet phases with direct methods are given.
\end{abstract}

PACS numbers: $61.10 . \mathrm{Dp}_{\mathrm{p}}$

\section{Introduction}

In every textbook about X-ray structure analysis one can read sentences like this: If the phases of the structure factors are known, then the crystal structure is known, for one can compute the electron density $\rho(r)$ by the Fourier summation

$$
\rho(r)=\frac{1}{V} \sum_{h} F(h) \mathrm{e}^{-2 \pi \mathrm{i} h r}=\frac{1}{V} \sum_{h}|F(h)| \mathrm{e}^{\mathrm{i} \varphi(h)} \mathrm{e}^{-2 \pi \mathrm{i} h r} .
$$

However, the trouble is the lack of the detectors to be sensitive for phases, which means that only the moduli of the structure amplitudes $|F(\boldsymbol{h})|$ can be derived from the measured intensities and their phases $\varphi(h)$ are unknown. These facts are called the "phase problem of crystallography".

In order to get experimental information on the phases of diffracted waves the only thing would be to carry out an interference experiment. For instance, superimposing two coherent waves with amplitudes $A_{1}$ and $A_{2}$ and phases $\alpha_{1}$ and 
$\alpha_{2}$ the resultant intensity $I_{\text {res }}$ depends on the amplitudes of the individual waves and on their phase difference

$$
I_{\text {res }} \sim\left|A_{\text {res }}\right|^{2}=\left|A_{1} \mathrm{e}^{\mathrm{i} \alpha_{1}}+A_{2} \mathrm{e}^{\mathrm{i} \alpha_{2}}\right|^{2}=A_{1}^{2}+A_{2}^{2}+2 A_{1} A_{2} \cos \left(\alpha_{2}-\alpha_{1}\right) .
$$

This is the principle of interferometry and holography.

Interference of several coherent diffracted waves inside a crystal can be achieved with a so-called multiple-beam case. $N$-beam multiple diffraction occurs when $N-1$ sets of lattice planes are simultaneously brought into position to diffract the incident beam, i.e. $N-1$ sets of lattice planes simultaneously satisfy Bragg's diffraction condition. In other words, $N$ reciprocal lattice points, including the origin, are simultaneously on the surface of the Ewald sphere. Then all the diffracted wave fields interact with each other due to the fact that all the difference vectors of the reciprocal lattice $h_{i}-h_{j}$ of the excited Bragg waves terminate on the Ewald sphere. Interference effects change the intensity of each Bragg reflection. It is this intensity variation which gives the phase information.

Already in 1949 Lipscomb [1] and at the same time Fankuchen suggested to use the three-beam case for experimental phase determination. But no experimental results were reported. At the end of the 70's and in the 80 's the feasibility of this method was shown by several authors (references can be found in the review article of Chang [2]). For understanding the interaction between the diffracted waves inside the crystal, the dynamical theory for multiple $\mathrm{X}$-ray diffraction must be used. For basic discussions we shall concentrate in this article on the three-beam case. We will give a brief survey of the dynamical theory for three-beam diffraction. The solution of the fundamental dynamical equations will be discussed with few mathematical and mostly physical arguments.

A systematically experimental way of generating multiple diffraction is an azimuthal scan, a so-called $\psi$-scan, around a scattering vector which is first aligned for reflection (primary reflection), i.e. it terminates on the Ewald sphere. By rotating the crystal in that way additional reciprocal lattice points can be brought on the Ewald sphere (secondary reflections). Thus, multiple diffraction is generated. If exactly one additional secondary reflection is excited then a three-beam case is generated.

\section{Three-beam interference}

This case is represented schematically in Fig. 1. Figure 1a shows the three-beam diffraction in crystal space, Fig. 1b - in reciprocal space (Ewald construction). The incident beam is diffracted at the lattice planes denoted by their reciprocal lattice vector $h$ and simultaneously at the lattice planes $g$. Thus, three strong waves are excited in the crystal: the incident wave and the two Bragg reflections $h$ and $g$. If we look, for example, for the waves propagated in the direction of the $h$-reflection, denoted by $K(h)$, then a superposition of two waves occurs: first, the directly diffracted wave at the lattice planes $h$ and second, a so-called Umweg-wave, which is twice reflected at the lattice planes $g$ and once more at the lattice planes $h-g$ into the direction of the $h$-reflection. These lattice planes $h-g$ must exist, since $h-g$ is also a vector of the reciprocal lattice (see Fig. 1b). $\pm(\boldsymbol{h}-\boldsymbol{g})$ 

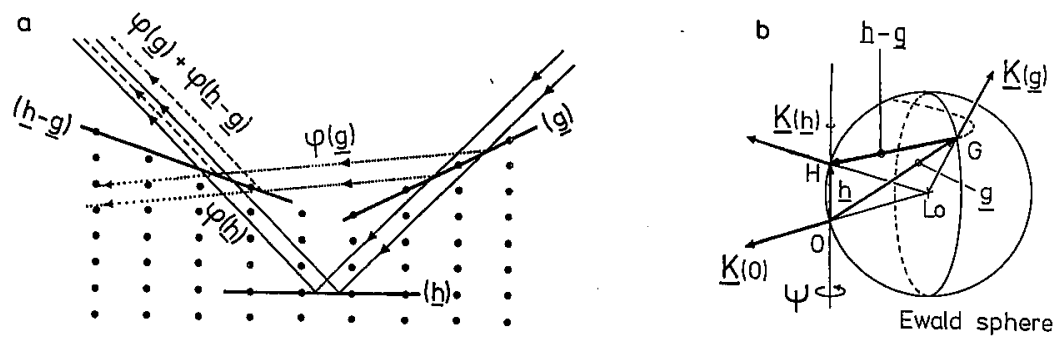

Fig. 1. Schematical representation of a three-beam case: (a) in crystal space, (b) in reciprocal space (Ewald construction).

couples the $h$ - and the $g$-reflection. However, let us neglect for a moment that the $\boldsymbol{h}$-reflection is also diffracted into the $\boldsymbol{g}$-reflection by $\boldsymbol{g}-\boldsymbol{h}$. Generally speaking, the wave vectors of the three beams do not lie in one plane as it is drawn in Fig. 1 for the sake of simplicity.

Renninger [3] has already proven experimentally that such an Umweg-wave must exist. He observed the intensity of the forbidden (222)-reflection of diamond during a $\psi$-scan around (222). Each time when a second reciprocal lattice point passed the Ewald sphere more or less strong diffracted intensity was measured, otherwise the intensity was very weak. Renninger called this effect Umweg-Anregung (Umweg excitation).

What is the phase difference between the primary reflection and the Umweg-reflection, which governs the resultant intensity due to interference (cf. Eq. (2))? The primary $h$-reflection has the phase $\varphi(h)$ due to the phase of its structure factor $F(h)$. The Umweg-wave has the phase $\varphi(g)+\varphi(h-g)$ due to the structure factor product $F(g) F(h-g)$ because it is twice diffracted at the corresponding lattice planes. Thus, the phase difference is given by

$$
\Phi_{3}(\boldsymbol{h}, \boldsymbol{g})=\varphi(g)+\varphi(h-g)-\varphi(h) .
$$

In the absence of anomalous dispersion effects Eq. (3a) can be rewritten

$$
\Phi_{3}(h, g)=\varphi(g)+\varphi(h-g)+\varphi(-h) .
$$

$\Phi_{3}(h, g)$ is a so-called triplet phase relationship.

As we shall see in the next Section, the dynamical theory of multiple-beam diffraction gives an additional phase shift, since in that regime Bragg reflection must be regarded as a spatial resonance phenomenon. Therefore, we must take a look at the dynamical theory for three-beam interference in order to understand the intensity variations during a $\psi$-scan scanning through a three-beam diffraction position.

\section{Three-beam dynamical theory}

The fundamental equations of the dynamical theory (5) are the solution of Maxwell's equations taking into account that the dielectric susceptibility has to be taken as translationally symmetric because of the short wavelength of X-rays in the order of atomic solution. Thus, all the wave fields show also translational 
symmetry and they have to be expanded in Fourier (Bloch) sums over all reciprocal lattice vectors. For example, the dielectric displacement $D$ is given by

$$
D(r)=\sum_{n} D(n) \exp [2 \pi \mathrm{i} K(n) r] \text { with } K(n)=K+n,
$$

where $n$ are the reciprocal lattice vectors. Analogous sums must be taken for the magnetic and electric field.

Substituting these sums into Maxwell's equations one gets the fundamental equations of dynamical theory [4]:

$$
D(n)=R(n) \sum_{m \neq n} \chi(n-m) D_{n}(m)
$$

$\chi(n)=\Gamma F(n) ; n, m$ run over all the reciprocal lattice vectors. $D(n)$ denotes the dielectric displacement of the wave field due to scattering vector $n . D_{n}(m)$ stands for the projection of $D(m)$ on $D(n)$, both are always perpendicular to their wave vectors $K(n)$ and $K(m)$, respectively. $F(n)$ is the structure factor. $\Gamma=\left(e^{2} / m_{\mathrm{e}} c^{2}\right)\left(\lambda_{0}^{2} / \pi V_{\mathrm{c}}\right)$, where $m_{\mathrm{e}}$ - electron mass, $V_{\mathrm{c}}-$ volume of the unit cell, is a small number of the order of $10^{-7}$ and gives the strength of the coupling between X-ray and electrons in the framework of Thomson's scattering theory.

The physical content of Eq. (5) can be described as follows. Each wave field $D(m)$ scatters part of its amplitude into the wave field $D(n)$ caused by the diffraction at the lattice planes $n-m$. The strength of the interaction between $D(m)$ and $D(n)$ is given by $\Gamma F(n-m)$. The resultant amplitude of the wave field $D(n)$ is given by the interference of contributions $D_{n}(m)$ of all excited wave fields $D(m)$.

The excitation strength of the different wave fields $D(n)$ is governed by the excitation error (Resonanzfehler)

$$
\begin{aligned}
& R(n)=B(n)^{-1}=K(n)^{2} /\left(K^{2}-K(n)^{2}\right), \\
& |K|=s_{0}\left(1-\frac{1}{2} \Gamma F(0)\right)=1 / \lambda, \quad s_{0}=1 / \lambda_{0} .
\end{aligned}
$$

$\lambda_{0}, \lambda$ are the wavelengths of vacuum and medium (crystal), respectively. This means that only such wave fields are strongly excited and have to be taken into account, which fulfil Bragg's law. Then the length of the wave vector $K(n)$ is equal to the radius of the Ewald sphere $K$ and $R(n)$ has its maximum value. $R(n)$ decreases with the distance of the end point of $n$ and $K(n)$ from the Ewald sphere, since $K(n)=K+n$.

This is the reason why it is allowed to cut off the infinite number of fundamental equations to three equations in the three-beam case, when only three strong waves $D(0), D(h)$ and $D(g)$ are simultaneously excited.

To make things simpler, for the calculation of the amplitude of the primary reflection $D(h)$, we set all the scalar products, which take into account the projection $D_{n}(m)$, equal to one. Therefore, we neglect the coupling between the $\pi$ and $\sigma$ polarization components of each wave field [5] and we are left with the following simplified system of equations for the three-beam case:

$$
\left|\begin{array}{lll}
B(0) & \chi(-h) & \chi(-g) \\
\chi(h) & B(h) & \chi(h-g) \\
\chi(g) & \chi(g-h) & B(g)
\end{array}\right|\left|\begin{array}{l}
D(0) \\
D(h) \\
D(g)
\end{array}\right|=0 .
$$


To solve these equations for the ratio $D(h) / D(0)$ the Bethe approximation is employed. This means the amplitude of $D(g)$ is expressed in terms of $D(0)$ and $D(h)$ using the third equation of (7). Substituting in the second equation of (7), for instance, and solving for $D(h) / D(0)$ we get

$$
D(h) / D(0)=R(h) \Gamma[F(h)+\Gamma R(g) F(g) F(h-g)] .
$$

This result can be interpreted as follows. The amplitude in the two-beam case, i.e. no secondary reflections are excited, given by $D_{2}(h) / D(0)=R(h) \Gamma F(h)$ (first term of (8)) is modified by higher-order terms due to excitation of other reflections (second term of (8)). Obviously, if $R(h)$ is negligibly small, i.e. Bragg's law for the $h$-reflection is not fulfilled, then no intensity can be observed in the direction of $\boldsymbol{K}(h)$. This is also true, even though other wave fields are excited by carrying out a $\psi$-scan around $h$, so that other reciprocal lattice points must pass through the Ewald sphere. In this case Bragg's law for the scattering of the secondary $g$-reflection into the $h$-reflection is not fulfilled, because the end points of the coupling vector $\boldsymbol{h}-\boldsymbol{g}$ does not terminate on the Ewald sphere.

Thus, a strict prerequisite in order to observe the modification of the intensity of the $h$-reflection by the additional excitation of other reflection is to keep $h$ precisely on the Ewald sphere during the $\psi$-scan.

In this case the primary $h$-reflection can be considered as a reference beam modulated by the secondary reflection, i.e. the $\psi$-scan experiment generating multiple diffraction closely resembles holography. The interference contrast gives the phase information. Therefore, the amplitude (intensity) of the primary $h$-reflection is normalized and (8) is rewritten

$$
D(h) / D(0)=R(h) \Gamma F(h)\{1+\Gamma R(g)[F(g) F(h-g) / F(h)]\} .
$$

Equations (8) and (9) confirm the considerations of Sec. 2. In the three-beam case the wave field $D(h)$ is given by a superposition of two waves: the directly diffracted wave governed by the structure factor $F(h)$ and the Umweg wave governed by the product of structure factors $F(g) F(h-g)$. As can be seen from Eq. (9) (cf. the [-]-term) the phase relationship which governs the interference of both waves is given by Eq. (3).

As can further be seen from (8) and (9) the resonance term (excitation error) $R(g)$ governs the amplitude of the Umweg wave and causes an additional phase shift by $180 \mathrm{deg}$ during the $\psi$-scan. Let us discuss this point in more details assuming that the $\psi$-scan is carried out in such a way that the end point of $g$ passes the Ewald sphere from inside to outside. At the beginning of the $\psi$-scan when $g$ terminates inside, it is $|\boldsymbol{K}(\boldsymbol{g})|<|\boldsymbol{K}|$, since $\boldsymbol{K}(\boldsymbol{g})=\boldsymbol{K}+\boldsymbol{g}$ (cf. Fig. 2a), i.e. the denominator of $R(g)$ is positive. When $g$ approaches the Ewald sphere, $R(g)$ gets larger and larger since the denominator approaches to zero, i.e. the amplitude of the Umweg wave increases. It has its maximum value when $g$ exactly lies on the Ewald sphere. When $g$ leaves the Ewald sphere, the amplitude of the Umweg wave decreases and $R(g)$ has changed its sign, since $|K(g)|>|K|$ when $g$ terminates outside (cf. Fig. 2b). Changing its sign $R(g)$ causes an additional phase shift by $180 \mathrm{deg}$. The behaviour of the amplitude and the phase shift of the Umweg wave during the $\psi$-scan is depicted in Figs. $3 \mathrm{a}$ and $3 \mathrm{~b}$. 


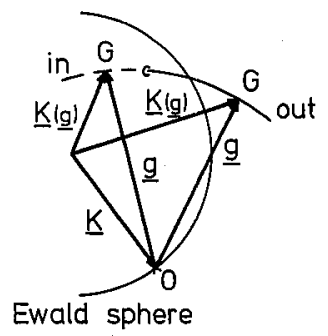

Fig. 2. Behaviour of $\boldsymbol{K}(g)$ during the $\psi$-scan from inside to outside.

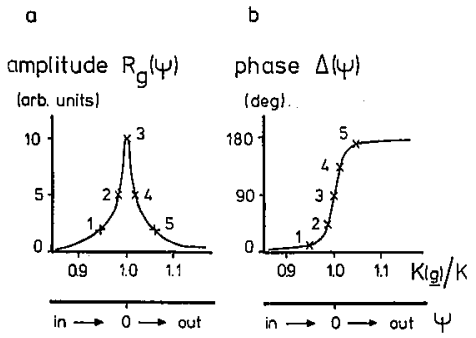

Fig. 3. Behaviour of the Umweg wave: (a) amplitude, (b) resonance phase shift.

Thus, the total phase relationship between the primary reflection and the Umweg reflection depends on $\psi$ and is given by

$$
\Phi_{\text {tot }}(\psi)=\Phi_{3}+\Delta(\psi)=\varphi(g)+\varphi(h-g)-\varphi(h)+\Delta(\psi) .
$$

$0 \leq \Delta(\psi) \leq 180^{\circ}$ for a $\psi$-rotation sense: inside $\rightarrow$ outside.

That is all we need to discuss the three-beam $\psi$-scan profiles for different triplet phase relationships.

\section{The $\psi$-scan profiles}

\subsection{Ideal profiles}

As already discussed above, a basic requirement for multiple-beam $\psi$-scans is that the end point of the primary scattering vector $h$ remains exactly on the surface of the Ewald sphere. Then the primary diffracted wave serves as a reference wave which amplitude remains constant, if no secondary reflections are excited. It is modulated by the Umweg waves due to the excitation of secondary reflections. This modulation gives information on the triplet phase relationship in case of three-beam diffraction.

As we have seen, it is absolutely necessary to know the rotation sense of the $\psi$-scan experiment. To be clear we discuss the $\psi$-scan profiles in case of a rotation sense when the secondary scattering vector $g$ passes the Ewald sphere from inside to outside. In the plots $\psi=0$ gives the exact three-beam position: $\psi \leq 0$ means $g$ terminates inside and $\psi \geq 0$ means $g$ terminates outside. 
Suppose the triplet phase of a three-beam case $0, h, g$ is zero: $\Phi_{3}=0^{\circ}$. Then, at the beginning of the $\psi$-scan $\Delta(\psi)=0$ and $\Phi_{\text {tot }}=0$. The amplitude of the Umweg wave is very small and the two-beam intensity $\left|D_{2}(h)\right|^{2}$ is observed. Scanning towards the three-beam position the amplitude of the Umweg wave increases. The primary wave and the Umweg wave interfere in a constructive way which leads to an increase in the resultant amplitude of $D(h)$. Thus, its intensity is increased. Very near to the three-beam position $\Delta(\psi)$ shifts very rapidly from 0 to $180^{\circ}$, then $\Phi_{\text {tot }}=180^{\circ}$. That means the interference becomes destructive and the intensity is decreased. At the end of the $\psi$-scan when the amplitude of the Umweg wave gets smaller and smaller the two-beam intensity is observed again. This $\psi$-scan profile for $\Phi_{3}=0$ is shown in Fig. 4a.

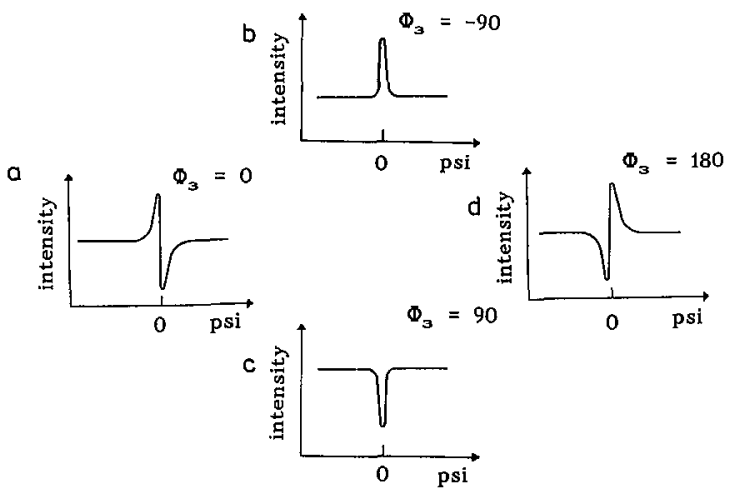

Fig. 4. Ideal three-beam diffraction profiles.

For $\Phi_{3}=180$ the $\psi$-scan profile is inversed with respect to $\psi=0$ as shown in Fig. 4d. This behaviour is explained as follows. At the beginning of the $\psi$-scan the phase relationship between the interfering waves $\Phi_{\text {tot }}=\Phi_{3}+\Delta(\psi)=180+0=$ 180. Thus, increasing the amplitude of the Umweg wave by scanning towards the three-beam position the resultant intensity is first decreased because of destructive interference and then increased. Because, if $\boldsymbol{g}$ terminates outside the Ewald sphere then in that case $\Phi_{\text {tot }}=180+180=0 \bmod 360$, which leads to constructive interference.

It follows the explanation of the three-beam profiles for $\Phi_{3}=+90$ or -90 . In the case of $\Phi_{3}=+90, \Phi_{\text {tot }}$ shifts from 90 to 270 during the $\psi$-scan. At the exact three-beam position $\Phi_{\text {tot }}=90+90=180$, since $\Delta(\psi)=90$ at $\psi=0$ as can be seen in Fig. $3 \mathrm{~b}$ and at the same time the amplitude of the Umweg wave has its maximum value. The result is a symmetrical profile around $\psi=0$ caused by destructive interference, since $\cos \Phi_{\text {tot }}$ is negative for $\Phi_{\text {tot }}$ in the range between 90 and 270 . In the case of $\Phi_{3}=-90, \Phi_{\text {tot }}$ shifts from +90 to -90 . Since at the exact three-beam position $\Phi_{\text {tot }}=-90+90=0$, a symmetrical profile is observed where the two-beam intensity is increased because of constructive interference, since $\cos \Phi_{\text {tot }}$ is positive for $\Phi_{\text {tot }}$ in the range between -90 and +90 . Both cases are depicted in Fig. $4 \mathrm{~b}$ and $4 \mathrm{c}$. 
The three-beam diffraction $\psi$-scan profiles for triplet phases of $+45,+135$, -45 and -135 must be between both the profiles of 0 and 90,90 and 180, 0 and $-90,-90$ and 180 , respectively. For example, the three-beam profile for +45 must first show a slight increase ( $\cos 45>0)$ and then a distinct decrease since in the exact three-beam position $\left(\Delta(\psi)=90, \Phi_{\text {tot }}=135\right)$, when the amplitude of the Umweg wave is highest, it results in a destructive interference $(\cos 135<0)$. All the ideal $\psi$-scan profiles are depicted schematically in Fig. 4a-d.

Hümmer and Billy [5] explained the ideal $\psi$-scan profiles due to three-beam interference effects by means of phase-vector diagrams.

\subsection{Profiles with Umweganregung and Aufhellung effects}

These ideal profiles can only be observed if the amplitudes of the interfering waves fulfil certain conditions. In general, the $\psi$-scan profiles consist of two parts: a phase-dependent part (ideal profile) due to the interference effect which bears the phase information and a symmetric phase-independent part due to the mean energy flow in a three-beam case which must be either incoherently added in case of Umweganregung effects or substracted in case of Aufhellung effects [6].

Remember the fundamental equation of interference (2). The first two terms represent the incoherent addition of the intensities of each wave. The third term represents the interference effect. If, for example, $A_{1} \gg A_{2}$ then the interference contrast is small compared to the phase independent intensity $A_{1}^{2}+A_{2}^{2}$. A similar effect occurs if one of the $\boldsymbol{h}$ - or $\boldsymbol{g}$-reflection is much more stronger or weaker than the other.

Suppose the $h$-reflection is very weak. This is similar to the original Renninger experiment [3]. Then, independent of the value of the triplet phase $\Phi_{3}$, intensity of the $g$-reflection is coupled into the $h$-reflection via $h-g$. This would lead to strong Umweganregung effects which give no phase information like in the Renninger experiment. On the other hand, suppose the $h$-reflection is strong, then intensity is coupled out off the $h$-reflection via $g-h$ into the $g$-reflection. This would lead to Aufhellung effects independent of the triplet phase. The ideal profiles can only be observed when the energy flow between the $h$-reflection and the $g$-reflection is well balanced, i.e. the same amount of energy is coupled out off and coupled into the $h$-reflection and $g$-reflection, respectively. This energy flow is governed by the law of conservation of energy. It can be described by a coupled system of differential equations [7]. It depends only on the moduli of the structure factors involved in a three-beam case.

Existence of such Umweganregung and Aufhellung effects were proved theoretically and experimentally by Weckert and Hümmer [6] and Hümmer et al. [8]. A theoretical example is shown in Fig. 5. The $\psi$-scan profiles are numerically calculated solving the fundamental equations of the three-beam dynamical theory where the triplet phase involved is set to $\pm 90 \mathrm{deg}$. The criterion whether Umweganregung or Aufhellung occurs is the ratio $Q=|F(g) F(h-g)| /|F(h)|^{2}$. Ideal profiles occur for $Q \approx 4$ (Fig. 5a). The profiles in Fig. 5b were calculated increasing $|F(g)|$ from 20 to 75 leaving the other structure factor moduli constant, i.e. $Q$ was increased to $\approx 13$. As a result strong Umweganreging effects occur, so that the destructive 

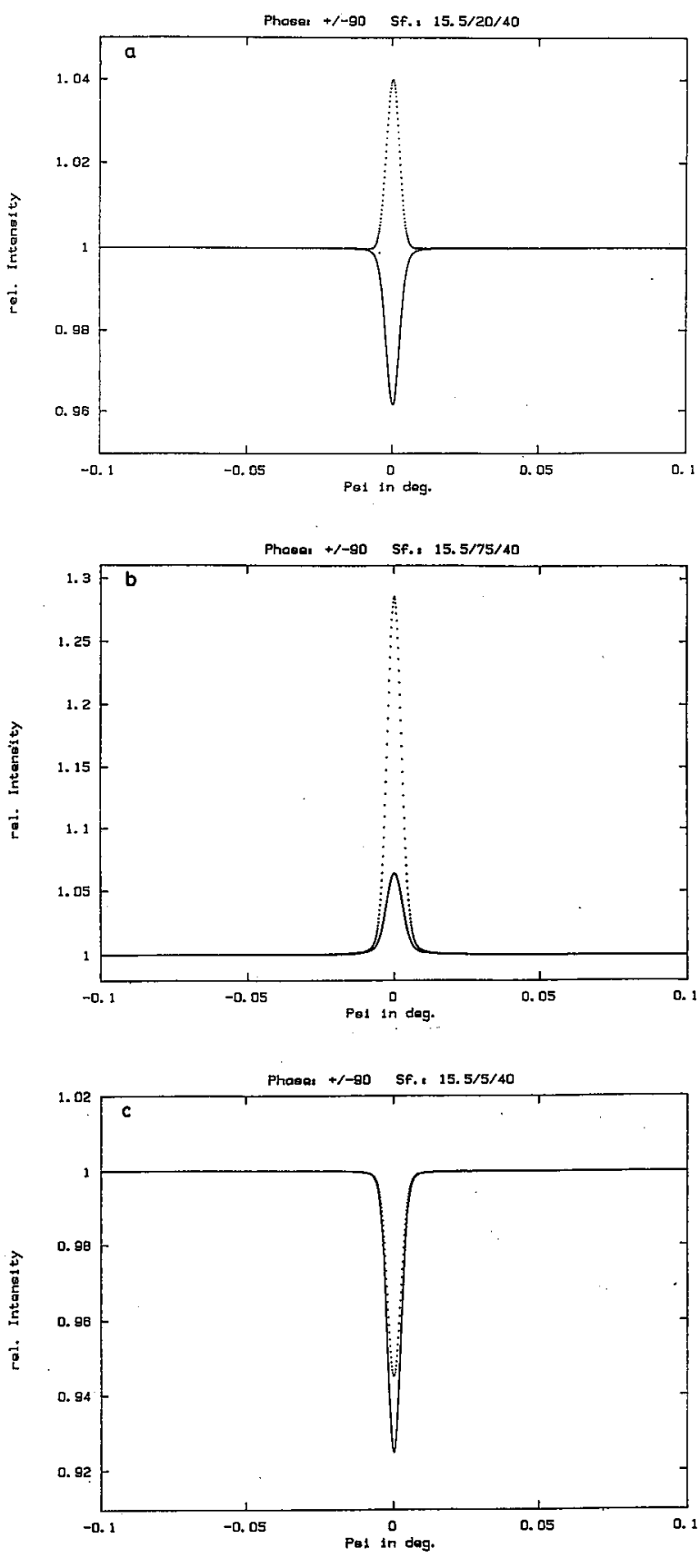

Fig. 5. Influence of the structure factor moduli on Umweganregung and Aufhellung, calculations by dynamical theory (solid: $\Phi_{3}=+90$, dotted: $\left.\Phi_{3}=-90\right)$ : (a) $|F(h)|=$ 15.5, $|F(g)|=20,|F(h-g)|=40 ;(b)|F(h)|=15.5,|F(g)|=75,|F(h-g)|=40$;

(c) $|F(h)|=15.5,|F(g)|=5,|F(h-g)|=40$. 
interference effect in case of +90 is overcompensated. But nevertheless, there is a big difference between both profiles. The relative intensity change of the two-beam intensity come to $+28 \%$ for $\Phi_{3}=-90$ and $6 \%$ for $\Phi_{3}=+90$. This means that an interference effect of $\pm 11 \%$ is superimposed by an phase-independent Umweganregung of $+17 \%$. The profiles in Fig. $5 \mathrm{c}$ were calculated decreasing $|F(g)|$ to 5 , i.e. $Q \approx 0.9$, leaving all the other parameters constant. As a result strong Aufhellung effects occur coming to $-7.5 \%$, the interference effect being $\pm 1 \%$. In the case that the Umweganregung or Aufhellung effects are dominating compared to the interference effect no phase information can be deduced.

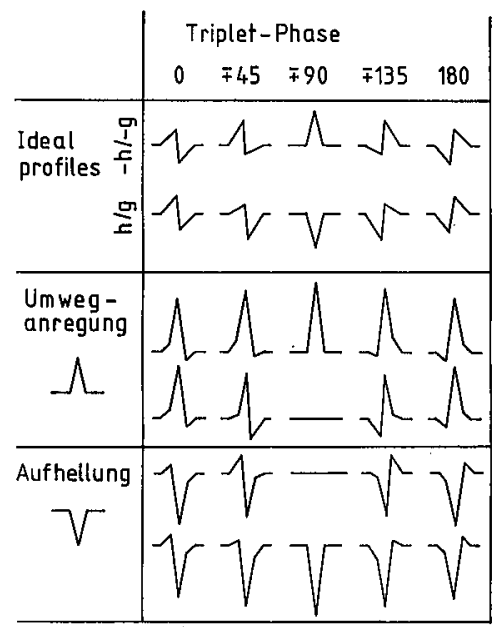

Fig. 6. Catalogue of possible $\psi$-scan profiles in case of three-beam diffraction.

Possible $\psi$-scan profiles are listed in Fig. 6. The profiles with Umweganregung or Aufhellung effects are gained adding a phase-independent symmetrical Umweganregung or Aufhellung profile to the ideal $\psi$-scan profiles. In Fig. 6 the magnitude of the Umweganregung and Aufhellung effects are chosen in such a way that the constructive or destructive interference effect is just compensated. As already discussed above the phase-independent parts may even overcompensate the interference effects (see Figs. $5 \mathrm{~b}$ and $5 \mathrm{c}$ ). This phase-independent part can be experimentally evaluated by comparing the $\psi$-scan profiles of the two centrosymmetrically related three-beam cases $0 / h / g$ and $0 /-h /-g$ where the structure factor moduli remain constant, i.e. the phase-independent parts are equally strong. However, the signs of the structure factors are reversed for both cases and therefore the sign of the triplet phase involved is also reversed.

It can be seen that in these cases phase determination is possible with an accuracy of about $45 \mathrm{deg}$ since all the $\psi$-scan profiles show significant differences. Moreover, also the sign of the triplet phases of non-centrosymmetric structures can be determined. This is extremely important for solving the enantiomorphy problem in light atom structures where anomalous dispersion effects are very weak. By determination of the sign of a triplet phase the absolute configuration is absolutely 
fixed. It should be pointed out that for our method no anomalous dispersion effects are needed.

\section{Experimental}

\subsection{The $\psi$-circle diffractometer}

It is known that the angular width of the $\psi$-scan profiles is of the order of some arc minutes. Moreover, the intensity change due to the three-beam interference is only a few percent, when the moduli of the structure factors involved have approximately the same magnitude in order to avoid strong phase-independent Umweganregung or Aufhellung effects. Therefore, the measurement of $\psi$-scan profiles rèquires high precision of the angular resolution and high accuracy of the $\psi$-scan. In any case, any staggering motion of the primary scattering vector must be avoided during the $\psi$-scan. That means, it must always be kept exactly on the Ewald sphere. As to our experimental experience exact $\psi$-scans are difficult to perform with an conventional four-circle diffractometer. Therefore, a special $\psi$-circle diffractometer was constructed (Fig. 7). This instrument has two perpen-

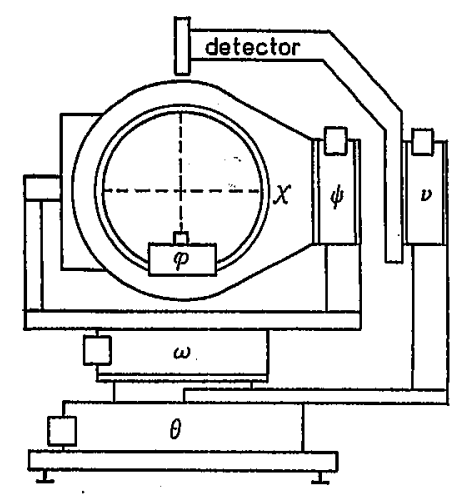

Fig. 7. Schematical drawing of the $\psi$-circle diffractometer. During the measurement of the three-beam $\psi$-scan profile the detector is set in the horizontal plane.

dicular circles denoted by $\vartheta$ and $\nu$ to move the detector. Four circles are available to move the crystal. The first crystal axis $\omega$ is parallel to the first detector axis $\vartheta$ ( $\omega-2 \vartheta$ relation). Perpendicular to the $\omega$-axis a second axis for the $\psi$-rotation is installed. This $\psi$-axis bears an Eulerian cradle with motions $\chi$ and $\varphi$. Thus an arbitrary scattering vector $h$ can be aligned with the $\psi$-axis and a $\psi$-scan can be performed by moving only one axis, namely the $\psi$-axis.

With the circles $\delta$ and $\nu$ the detector can be moved to any point on a hemisphere above the horizontal plane defined by the incident beam and the $\psi$-axis. In this way the $\psi$-angle of the exact three-beam position can be controlled measuring the profile of the second Bragg reflection by means of a $\psi$-scan. All circles are 
computer controlled and driven by stepper motors. The angular resolution is at least $0.001^{\circ}$.

\section{2. $X$-ray sources}

The properties of the three-beam diffraction profiles make high demands not only on the diffractometer, but also on the $\mathrm{X}$-ray sources.

The measured $\psi$-scan intensity profiles near a three-beam position are given by the convolution of the intrinsic three-beam profile which depends on the diffraction geometry and the moduli of the structure factors involved, with the experimental apparatus functions for divergence and wavelength bandwidth. Due to this convolution the interference contrast is higher for smaller divergence and bandwidth.

Another point which should be noticed is that even for a small molecule structures the sequence of three-beam positions on the $\psi$-scale are very dense. For example, Fig. 8 shows the dependence of $\psi$ positions on the wavelength for three-beam cases of L-asparagine monohydrate (volume of the unit cell $0.646 \mathrm{~nm}^{3}$ ) in a certain range. The thick straight line represents the three-beam case of interest

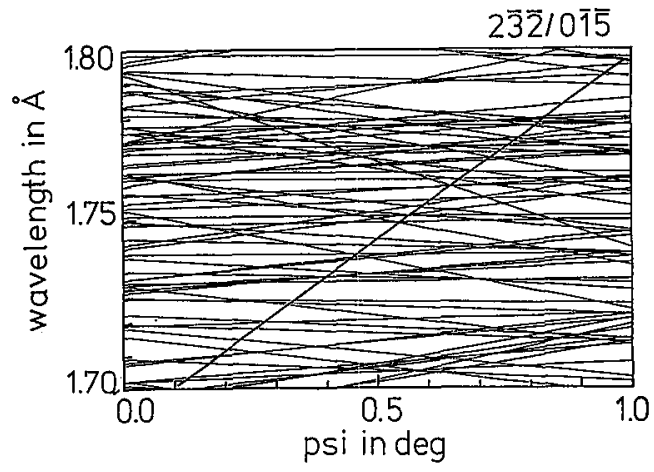

Fig. 8. Dependence of $\psi$ positions of multiple-beam points on wavelength for L-asparagine monohydrate.

$0 / h / g: h=(2-3-2), g=(0-1-5)$. All the thin straight lines represent other three-beam cases for the same primary reflection $h$, but which are not indexed. In order to avoid overlapping of the first three-beam case with other ones a proper wavelength must be chosen, so that that the angular distance on the $\psi$-scale is at least 0.1 degree. For the example shown in Fig. 8 this would be the case for $\lambda=0.173 \mathrm{~nm}$. For smaller divergence overlap is less likely as the interference profiles are less wide.

Therefore, for this type of experiments an X-ray source with high brilliance and tunable wavelength is necessary.

\subsubsection{Rotating anode}

For our in-house experiments a Rigaku high brilliance rotating-anode generator is available. We use a point focus of $0.3 \times 0.3 \mathrm{~mm}^{2}$ effective size with $5.4 \mathrm{~kW}$ 
load on a copper target. The radiation is filtered by a $100 \mu \mathrm{m}$ nickel foil ( $\beta$ filter), hence the spectrum consists mainly of the $K_{\alpha}$ emission lines. An appropriate divergence $\left(\sim 0.025^{\circ}\right)$ is achieved by a focus to crystal distance of $1.2 \mathrm{~m}$. Absorption in air between focus and crystal is reduced by an evacuated pipe.

The advantages of the rotating anode is a nearly unlimited availability and good beam stability. Disadvantages are the fixed wavelength and the relative low intensity due to the long distance needed to get a low divergence.

\subsubsection{Synchrotron radiation}

The advantages of synchrotron radiation (SR) are tunability of the wavelength and high brilliance. Most of our experiments were performed at HASYLAB in Hamburg using SR from a bending magnet of storage ring DORIS running at an electron energy of 3.7 to $4.5 \mathrm{GeV}$. Monochromatization is done by a computer controlled fixed-exit double crystal $\mathrm{Ge}(111)$ monochromator giving a bandwidth of approximately $0.01 \%$. We used wavelengths in the range from 0.07 to $0.25 \mathrm{~nm}$. The divergence at a photon energy of $8 \mathrm{keV}$ is about 0.008 degree. The diffracted intensity is about 100 times higher than the intensity from the rotating anode setup described above.

Disadvantages are beam instabilities which affect the experiment seriously and the limited amount of available beam time.

\subsection{Crystals}

The crystals we normally use for experimental phase determination were non-cut with grown faces. The crystal dimensions vary from 0.1 to $0.4 \mathrm{~mm}$. They were bathed in the incident beam.

It should be pointed out that the crystals need not be ideally perfect. Most of the crystals investigated showed some mosaicity, which can also be seen sometimes in the three-beam profiles. The mosaicity can be measured from the width of the two-beam profile with a highly collimated incident beam. As a rule, if the FWHH of the two-beam profile exceeds 0.1 degree using SR (divergence approximately 0.01 degree) then the crystal cannot be used for phase determination. However, most of the crystals show a mosaic distribution as shown in Fig. 15, i.e. they consist of several larger mosaic blocks which can be very well resolved. Such types of crystals can still be used for phase determination because the highly collimated SR allows to excite only one of the blocks, which serves then as a very good quality single crystal.

\subsection{Experimental results}

The theoretical results dicussed in the foregoing chapters were verified by several hundreds of experimental profiles using the rotating anode equipment or SR. In order to test the power of our method as a tool for quantitative triplet phase determination, we made measurements with several organic non-centrosymmetric small molecule structures with unit-cell volumes up to $3 \mathrm{~nm}^{3}$. The investigated structures are listed in Table 1 of the paper [8]. The result is that triplet phases can be experimentally determined from the three-beam diffraction profile with an 
accuracy of about 45 degrees - which means they can be assigned to phase octants with centres at $0 \bmod 45^{\circ}$ - in spite of Aufhellung and Umweganregung effects, provided that these phase-independent parts are not the dominant effects.

By visual evaluation nearly $95 \%$ triplet phases were determined correctly. About 5\% of the three-beam profiles showed irregularities incompatible with our catalogue in Fig. 6.

The $\psi$-scan profiles shown in the following refer to so-called "in-out" $\psi$-scans, i.e. for $\psi<0$ the second reciprocal lattice vector $g$ terminates inside the Ewald sphere, for $\psi>0$ it lies outside. $\psi=0$ marks the three-beam position. In each figure the indices of the primary $h$-reflection and the secondary $g$-reflection as well as the triplet phase calculated from the known structure are given.

\section{Applications}

\subsection{Determination of absolute structures}

The term "absolute structure" $[9,10]$ has different meanings dependent on the point group symmetry. It comprises the different ambiguities occuring for non-centrosymmetric structures. For enantiomorphic groups, containing only pure rotation axes, this ambiguity is called "absolute structure" for chiral structures and "absolute conformation" for achiral structures. For hemimorphic or polar point groups the structure has to be fixed with respect to the direction of the polar axis. For point groups containing rotoinversions like $\overline{3}, \overline{4}$ or $\overline{6}$ the structure has to be fixed with respect to an absolute assignment of the crystal axes.

The usual way to fix the absolute structure is the exploitation of anomalous dispersion effects. This can be done by refining an enantiomorph sensitive parameter [11] or by measuring the intensity of selected Bijvoet-Friedel pairs. Difficulties arise if there are only weak anomalous scatterers in the structure (e.g. light atom structures) or if the crystals have only very small size so that it is difficult to obtain good statistic for the reflection intensities necessary to exploit anomalous dispersion effects.

As pointed out above by three-beam diffraction also the sign of the triplet phase, defining its modulus in the range between 0 and $180^{\circ}$, can be determined. Since both absolute structures differ in the sign of their triplet phases the best selectors would be triplet phases near $90^{\circ}$. It should be mentioned that this method works without using anomalous dispersion effects, therefore it can be used in particular for light atom structures.

In Figs. 9 and 10 two examples are shown where we had both enantiomorphic forms available. The details about the structures are given in the figure captions. For these light atom structures determination of the absolute structure by means of anomalous dispersion effects would be difficult. It is obvious from the figures that the same three-beam case for two different enantiomorphic forms gives exactly the negative triplet phase. The noise in the profiles is mainly due to primary beam instabilities of the synchrotron source and not statistics.

Another example is shown in Fig. 11, where it was not possible to determine the absolute structure by means of anomalous dispersion effects using copper ra- 

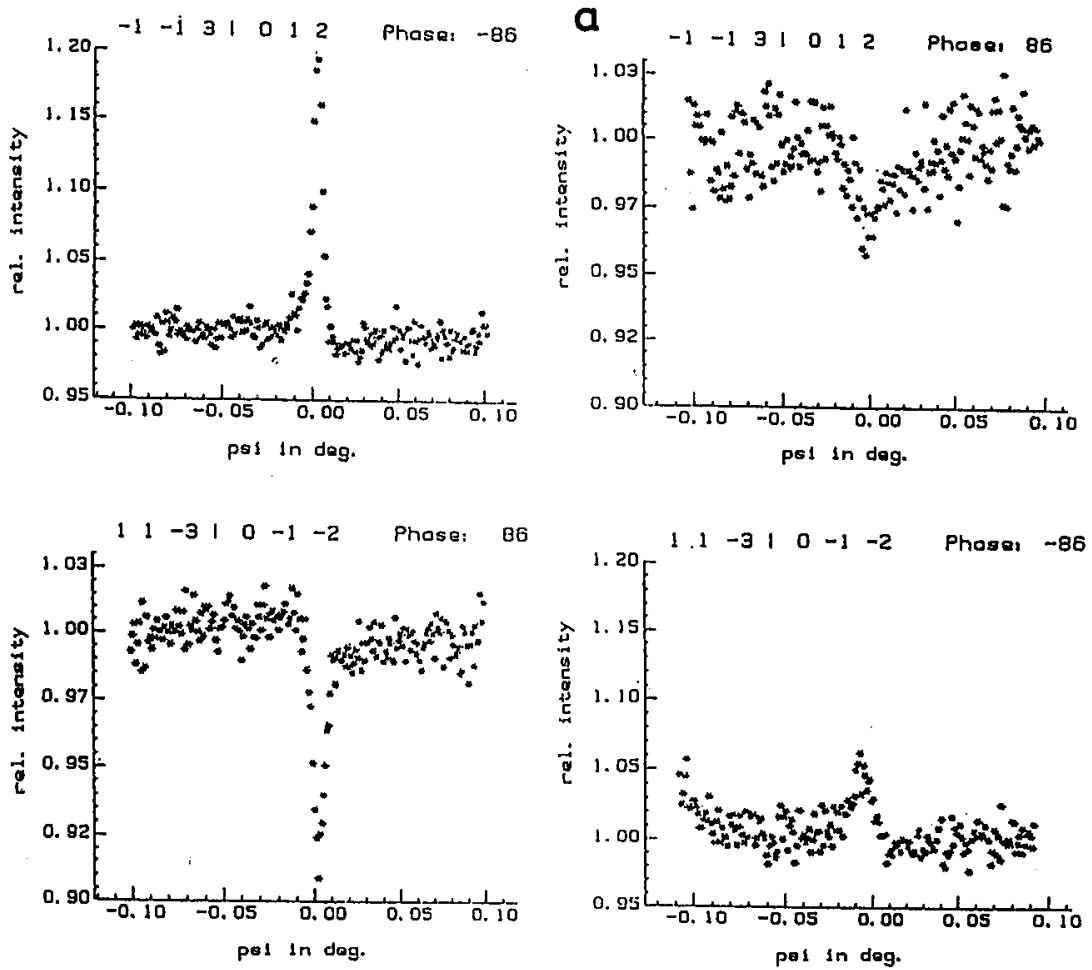

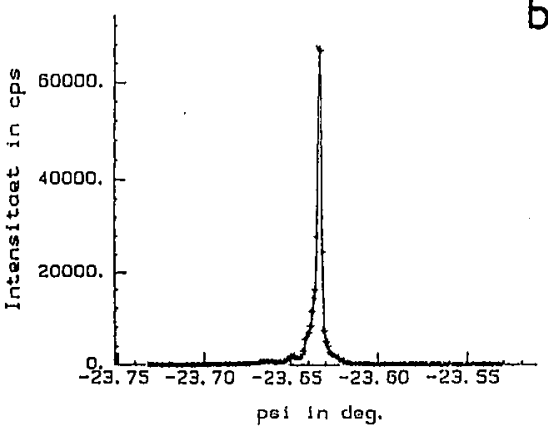

(+)Butafenon+Welnsăure

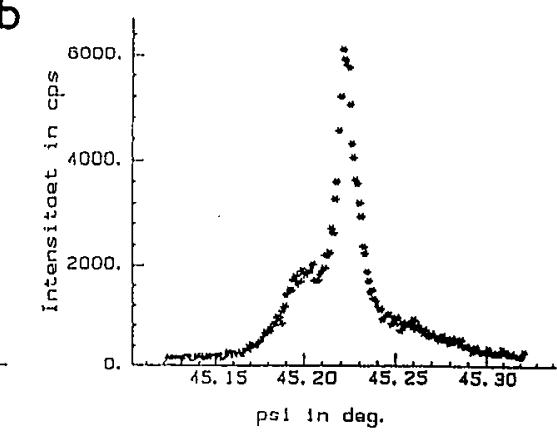

(-)Butafenon+Woinsăure

Fig. 9. (a) $\psi$-scan profiles of three-beam case $-1-13 / 012$ and its centrosymmetrically related one 11-3/0-1-2 of $(+)$ and $(-)$ Butafenon measured with synchrotron radiation at $\lambda=0.18 \AA$. (t)/(-)Butafenon: $P 2_{1}, a=7.51 \AA, b=9.16 \AA, c=20.85 \AA, \beta=95.2^{\circ}$, $Z=2\left(\mathrm{C}_{23} \mathrm{H}_{31} \mathrm{NO}_{3}+\mathrm{C}_{4} \mathrm{H}_{6} \mathrm{O}_{6}+\mathrm{H}_{2} \mathrm{O}\right)$. (b) $\psi$-scan profiles of the secondary reflections of the three-beam case shown in Fig. 9a. The mosaic spread of the (-)Butafenon crystal was much higher than the one of the $(+)$ Butafenon crystal. 

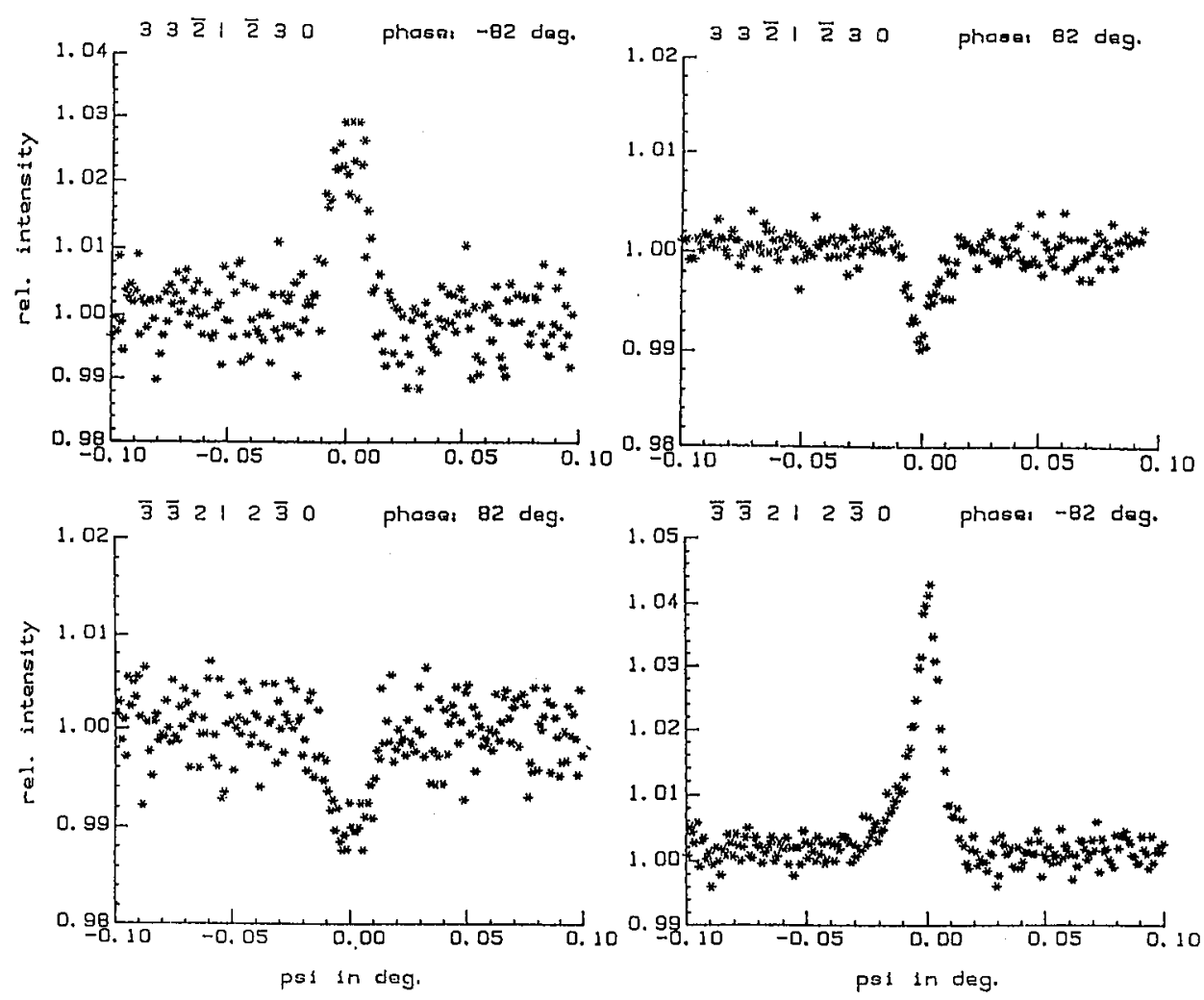

Fig. 10. $\psi$-scan profile of three-beam case $33-2 /-230$ and $-3-32 / 2-30$ of $(-)$ and (+)Rolipram measured with synchrotron radiation at $\lambda=2.3900 \AA .(+) /(-)$ Rolipram: $P 2_{1}, a=18.55 \AA, b=7.38 \AA, c=22.63 \AA, \beta=104.7^{\circ}, Z=8\left(\mathrm{C}_{18} \mathrm{H}_{21} \mathrm{NO}_{3}\right)$.

diation since the crystals were only available as very small needles. The mosaic spread of these crystals was rather small, therefore experimental phase determination worked very well.

\subsection{Future aspects: phase determination of large structures and structure solution}

Up to now most of the structures were solved by direct methods, which use statistical evaluation of triplet phases. Meanwhile, structures with approximately 200 independent non- $\mathrm{H}$ atoms can be solved more or less routinely. However, difficulties arise for larger structures. Therefore, the question should be answered whether it is possible to solve large structures by combination of measured triplet phases with direct methods.

The first step into this direction is to test whether it is possible to measure triplet phases of structures with large unit cell dimensions, where it is impossible to avoid overlapping of several three-beam cases. In preliminary experiments 

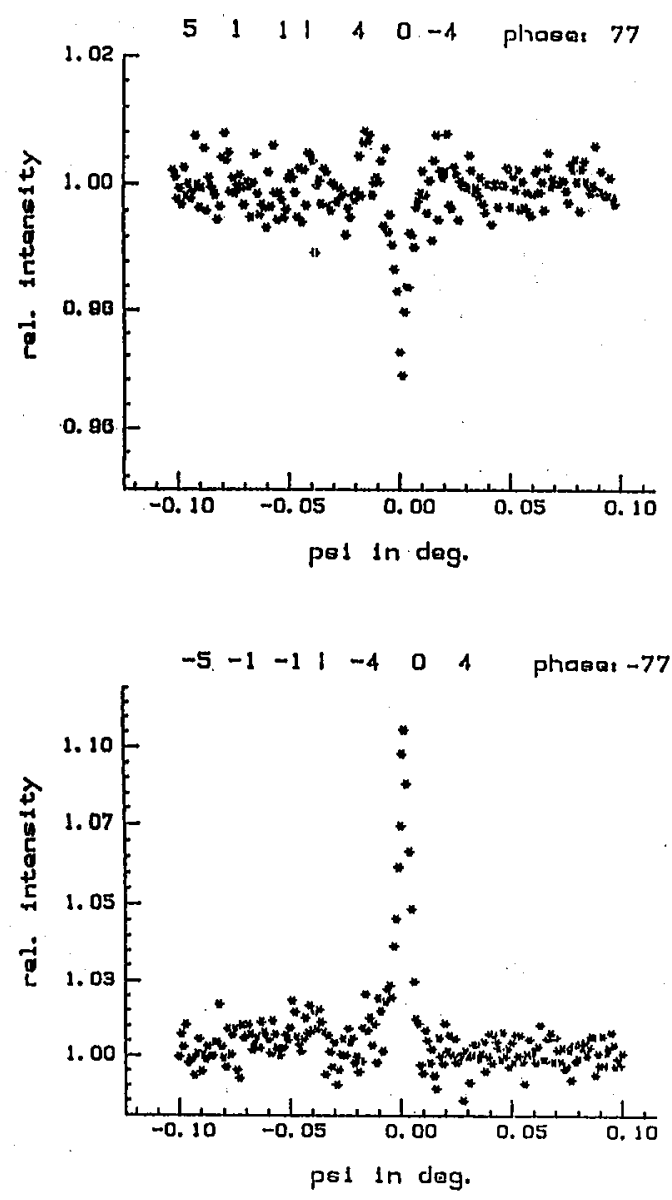

Fig. 11. $\psi$-scan profile of three-beam case $511 / 404$ of $\mathrm{C}_{22} \mathrm{H}_{23} \mathrm{O}_{7} \mathrm{~N}$ measured with synchrotron radiation at $\lambda=1.6527 \AA ; C 2, a=23.29 \AA, b=6.47 \AA, c=14.97 \AA$, $\beta=116.4^{\circ}$.

using small molecule structures we investigated the way in which $\psi$-scan profiles of three-beam cases with strong structure factors are influenced by overlapping three-beam reflections with weak structure factors. The structure factor moduli of the wanted three-beam cases were chosen to fulfil the following condition: $2 \leq Q(h g) \leq 6$ with $Q(h g)=F^{\prime}(g) F^{\prime}(h-g) / F^{\prime}(h)^{2}\left(F^{\prime}\right.$ are structure factor moduli corrected for polarization in case of using SR). It was found that in spite of overlap of other weak three-beam reflections with respect to the same primary reflection the triplet phase of the wanted three-beam case can be exploited provided that $Q(\boldsymbol{h} g)_{\text {weak }} \leq 0.1 Q(\boldsymbol{h g})_{\text {strong. }}$. Thus, one can be confident that experimental phase determination is also possible for large structures if the overlapping weak three-beam reflections fulfil this condition. 

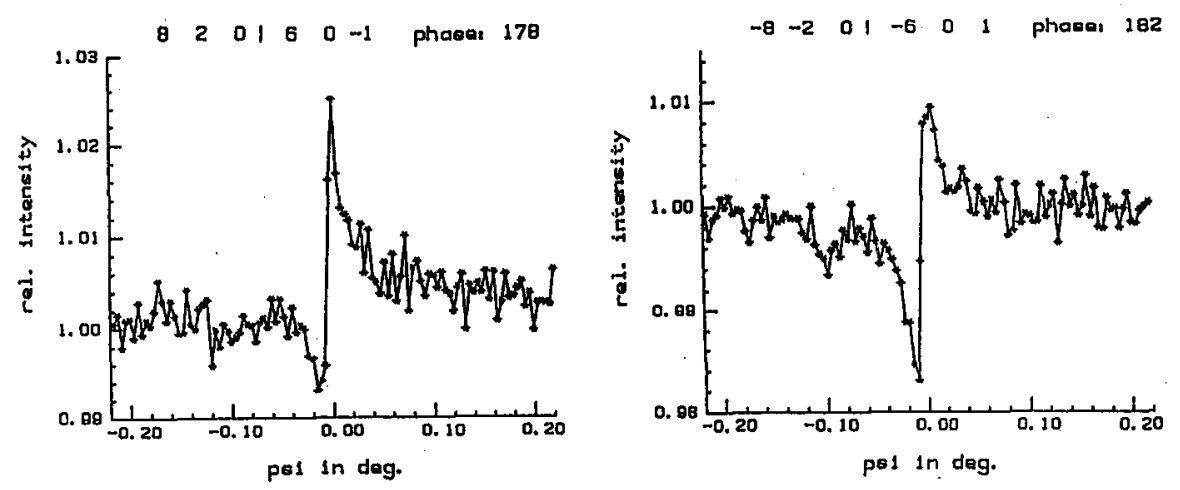

Fig. 12
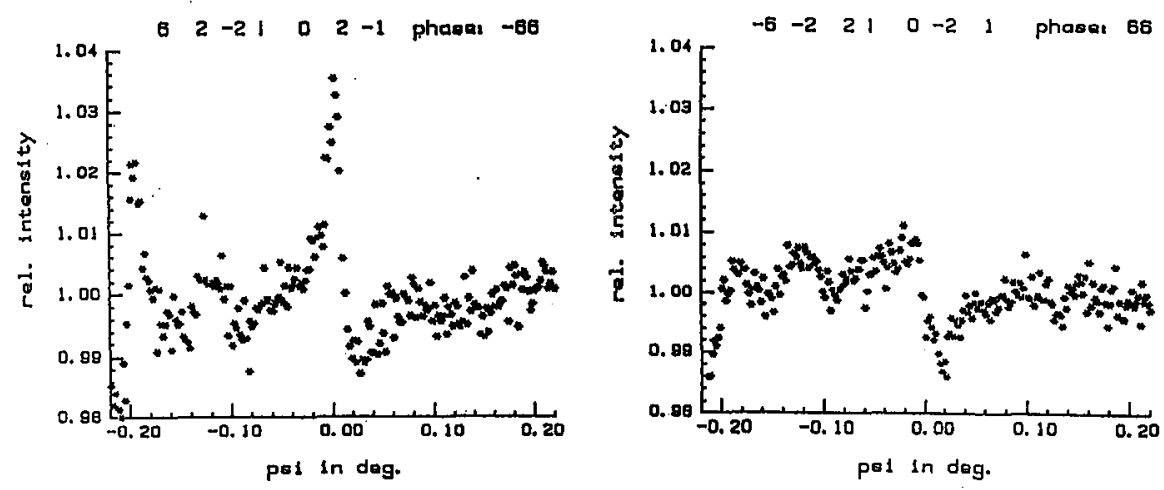

Fig. 13

Fig. 12. $\psi$-scan profile of three-beam case $820 / 60-1$ of Myoglobin, $\lambda=1.54 \AA$; Myoglobin: $P 2_{1}, a=64.51 \AA, b=30.91 \AA, c=34.86 \AA, \beta=105.8^{\circ}$.

Fig. 13. $\psi$-scan profile of three-beam case $62-2 / 02-1$ of Myoglobin, $\lambda=1.9349 \AA$.

In Figs. 12 to 14 the first triplet phases measured in small proteins are shown (see also [12]). In each of the diagrams about 200 to 300 weak three-beam cases occur in a $\psi$-angular range of $+/-0.1^{\circ}$ with respect to the three-beam position of the main three-beam case. In Fig. 15 the profile of a secondary reflection during a $\psi$-scan is shown. The crystal quality was rather bad, but due to the small divergence at the synchrotron we were able to select just one of the big mosaic blocks to perform the experiment.

As the structure factor moduli of the wanted three-beam case have to be strong in large structures, only the phases of low and medium resolution reflections are accessible by experimetal phase determination. 

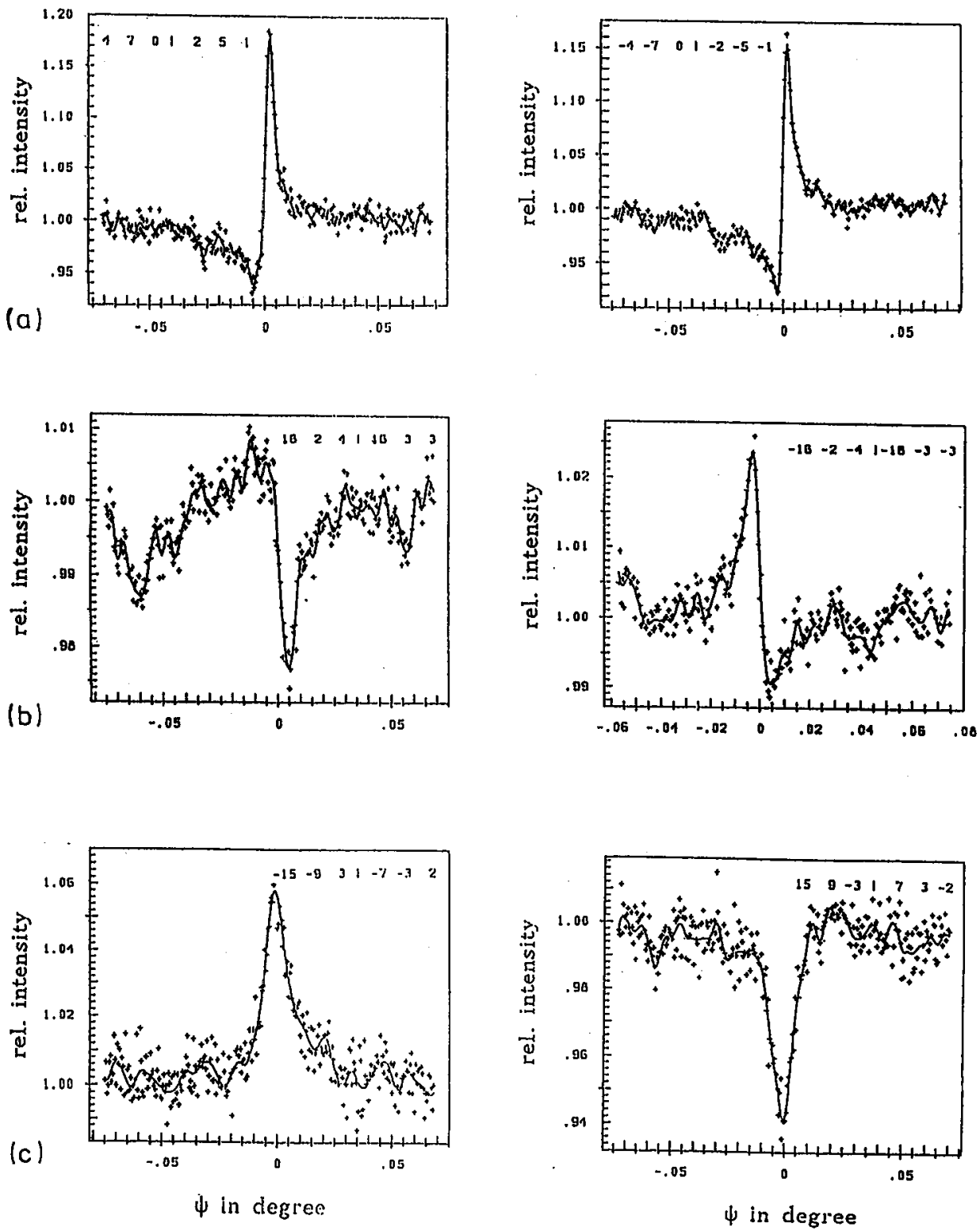

Fig. 14. $\psi$-scan profiles of Lysozym. Lysozym: $P 4_{3} 2_{1} 2, a=78.9 \AA, c=38.1 \AA ;$ (a) three-beam case $470 / 251, \lambda=1.5514 \AA, \Phi_{3}=180$; (b) three-beam case $1624 / 1633$, $\lambda=1.5433 \AA, \Phi_{3}=39$; (c) three-beam case $159-3 / 73-2, \lambda=1.5558 \AA, \Phi_{3}=72$. 


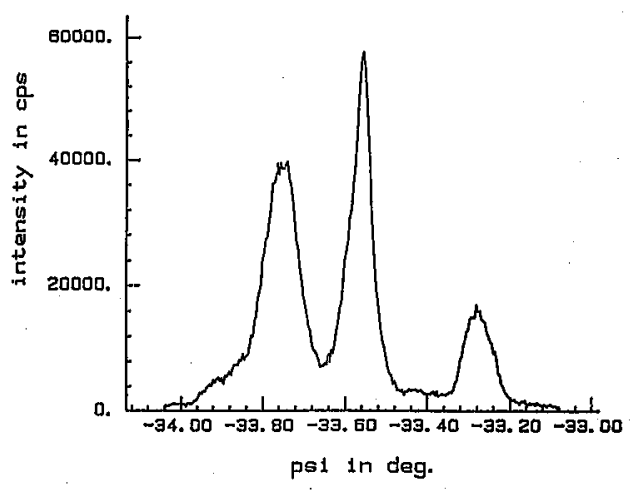

Fig. 15. Profile of the secondary reflection $60-1$ of the $\psi$-scan of Fig. 12. The interference experiments were performed with the mosaic block in the middle.

This work was funded by the German Federal Minister of Research and Technology under contract No. 05463 IXB5.

\section{References}

[1] W.N. Lipscomb, Acta Crystallogr. 2, 193 (1949).

[2] S.-L. Chang, Cryst. Rev. 1, 87 (1987).

[3] M. Renninger, Z. Phys. 106, 141 (1937).

[4] Z.G. Pinsker, in: Dynamical Scattering of $X$-rays in Crystals: Solid-State Sciences, Vol. 3, Eds. M. Cardona, P. Fulde, H.-J. Queisser, Springer-Verlag, Berlin 1978.

[5] K. Hümmer, H. Billy, Acta Crystallogr. A 42, 127 (1986).

[6] E. Weckert, K. Hümmer, Acta Crystallogr. A 46, 387 (1990).

[7] R.M. Moon, C.G. Shull, Acta Crystallogr. 17, 805 (1964).

[8] K. Hümmer, E. Weckert, H. Bondza, Acta Crystallogr. A 45, 182 (1989); ibid., 46, 393 (1990).

[9] P.G. Jones, Acta Crystallogr. A 42, 57 (1986).

[10] H. Burzlaff, K. Hümmer, Acta Crystallogr. A 44, 506 (1988).

[11] H.D. Flack, Acta Crystallogr. A 30, 569 (1974).

[12] K. Hümmer, W. Schwegle, E. Weckert, Acta Crystallogr. A 47, 60 (1991). 